\title{
Enhanced shRNA delivery by the combination of polyethylenimine, ultrasound, and nanobubbles in liver cancer
}

\author{
Hongbo Li ${ }^{\mathrm{a}, \mathrm{b}}$, Ziyu Wang ${ }^{\mathrm{a}}$, Jia Zhang ${ }^{\mathrm{b}}$, Chenyan Yuan ${ }^{\mathrm{b}}$, Hao Zhang ${ }^{\mathrm{b}}$, Xinxin Hou $^{\mathrm{b}}$ and \\ Dongsheng Zhang ${ }^{\mathrm{b}, *}$ \\ ${ }^{a}$ Department of Ultrasonography, The Affiliated Hospital of Nanjing University of Chinese Medicine, \\ Nanjing, China \\ ${ }^{\mathrm{b}}$ Jiangsu Key Laboratory for Biomaterials and Devices, Medical School, Southeast University, \\ Nanjing, China
}

\begin{abstract}
.
BACKGROUND: Traditional cancer treatments such as surgery, radiation, and chemotherapy destroy both cancer and normal cells, which limit their clinical application. It is difficult to achieve the best results for any liver cancer patients using any single treatment method. Gene therapy for HCC demands non-invasive, efficient, targeted and safe gene transfection strategies.

OBJECTIVE: In this study, a nonviral shRNA gene delivery system utilizing a combination of PEI, US, and NBs was developed for targeting survivin in liver Cancer.

METHODS AND RESULTS: The PEI-shRNA-NBs cumulated in the tumor tissue because of the EPR effect. By exposure to the US, micelles shRNA may be released from PEI-shRNA-NBs in tumor tissues and the shRNA then transmitted efficiently to cancer cells. Considerably enhanced therapeutic outcome was obtained with the gene silencing effect enhanced. CONCLUSIONS: PEI-shRNA-NBs possess the potential to become promising tools intended for shRNA delivery.
\end{abstract}

Keywords: Nanobubbles, transfection, shRNA, gene carrier, ultrasound

\section{Introduction}

Hepatocellular carcinoma (HCC) has a poor prognosis and the therapeutic options currently available are largely ineffective [1]. RNA interference (RNAi) is a biological pathway of gene silencing. Many studies have demonstrated the great potential associated with RNAi in HCC [2]. Despite the great potentials, significant medical effect hasn't been demonstrated. One major challenge is to deliver shRNA into cancer cells [3]. On account of the poor half-life of uncoated shRNA in the bloodstream and the widely existing RNAase, uncoated shRNA may be degraded quickly. Furthermore, large size shRNA and negatively charged backbone have low accumulation and are unable to enter the cell membrane [4].

Ultrasound (US)-mediated gene delivery (USMD) exhibits possibilities for increasing the efficiency of gene into tissues and cells, a technique generally known as sonoporation (the transient permeabilization of cell membrane) [5]. Nanobubbles (NBs) as cavitation nuclei oscillate and collapse under US

\footnotetext{
${ }^{*}$ Corresponding author: Dongsheng Zhang, School of Medicine, Southeast University, Nanjing, 210009, China. E-mail: zdszds1222@163.com.

0928-7329/19/\$35.00 (c) 2019 - IOS Press and the authors. All rights reserved

This article is published online with Open Access and distributed under the terms of the Creative Commons Attribution NonCommercial License (CC BY-NC 4.0).
} 
stimulation. Cell permeability can be altered and pores in the endothelial layer produced, which allow macromolecules to enter the target tissue [6]. As a physical transfer methodology, USMD methods have much preferred quality, include reduced toxicity, low immunogenicity, low invasiveness, organs targeted with high specificity, and broad usefulness [7].

NBs have been recommended as a prospective device for gene delivery [8]. Due to the large size, microbubbles cannot pass through the tumor vascular endothelium. NBs offer several advantages in targeted gene transfection. Due to the enhanced permeability and retention (EPR) effect, NB can accumulate in tumor tissue, which can increase cell permeability during US exposure. The sonoporation effects of NBs can also be detected under low frequency US exposure [9]. NBs has become a desirable tool for shRNA delivery.

Extracellular nucleases can degrade the released DNA at the target site and the cells are not efficient at taking DNA. NB therefore sends DNA with only a small amount of DNA uptake and expression. USMD combined with nonviral polycationic polymer carriers has also been used to enhance gene transfer efficiency. PEI is one of the most efficient non-targeted polycations utilized for gene delivery [10]. Acid-catalyzed polymerization of aziridine produces a highly branched network, which ensnares DNA. On account of the near neighborhood of numerous linker amino groups, PEI maintains a substantial buffering ability at almost any pH. Sonoporation can improve the efficiency of PEI [11].

Survival targeting strategies have become a promising strategy for HCC [12]. Steady survivin knockdown restricted HCC cell proliferation, increases sensitivity to apoptosis, imprisoned cell cycle in the G1 phase and also resulted in significant mitotic catastrophe. Additionally, cells stably expressing survivin shRNA in vivo study indicated that tumor growth is inhibited by spontaneous apoptosis of cancer cells and animal survival was significantly prolonged [13].

In this study, we evaluated the therapeutic potential of Polyethylenimine (PEI) conjugated shRNA with the use of nanobubbles (NBs) as carriers and ultrasound triggered release for enhanced delivery of complex to the cells for targeting anti-apoptosis gene survivin. Herein, we expect PEI-shRNA-NBs with effective shRNA protection and high transfection efficiency under US exposure, possess the potential to become promising tools intended for shRNA delivery.

\section{Methods}

\subsection{Materials}

Phospholipids including DSPC (1, 2-distearoyl-sn-glycero-3-phosphocholine), DSPE-PEG2000 (Distearoyl phosphoethanolamine-PEG2000) and DPPA (1,2-dipalmitoyl-sn-glycero-3-phosphate) were used in powdery form (Lipoid, Germany). Pluronic F-68 and bPEI (Branched polyethylenimine) from Sigma Aldrich. AnnexinV-FITC and PI from Key Gen Biotech (Nanjing, China). Dimethyl sulfoxide (DMSO) from Sigma (USA); Sulfur hexafluoride $\left(\mathrm{SF}_{6}\right)$ gas from the Specialty Gas plant.

\subsection{Preparation of $N B s$}

Chloroform: dimethyl carbinol (9-mL volume, 2:1, v/v) solution in a round-bottom flask were used to dissolve phospholipids (DSPC, DPPA and DSPE-PEG2000, molar ratio 80:12:8) dried by evaporation under reduced pressure at $50^{\circ}$ water bath until phospholipid membrane was formed. The membrane was then dissolved with $5 \mathrm{~mL}$ of liquid (10\% glycerol and Pluronic F-68). 
The air above the obtained liposomal suspension was substituted with replaced with $\mathrm{SF}_{6}$. The NBs were finally produced by intense shaking using a shaker. After low speed centrifugation, prepared bubbles were divided into two parts. After removing the bottom liquid layer, the NBs in the lower fraction were collected. The NBs concentration was determined using a hemacytometer.

\subsection{Preparation of PEI-shRNA-NBs}

The survivin-sh plasmid, encoding green fluorescence protein, was designed to target survivin gene. Survivin shRNA plasmid (the sequence 5/-GCCCAGTGTTTCTTCTGCT -3/) were synthesized in GenePharma Co. Ltd. Zero point five mg of shRNA and a designed amount of PEI was dissolved separately in PBS. Gel electrophoresis was performed to evaluate the shRNA condensation capability connected with PEI. PEI and shRNA were mixed together at various N/P ratios of PEI to $\operatorname{shRNA}(1: 4,1$ : $2,1: 1,2: 1,3: 1$, and $6: 1$ ). After the development of the polyplex micelle, the binding ability of PEI and shRNA was checked. PEI-shRNA-NBs complexes were prepared by surface conjugation. Morphological characteristics of the NBs and PEI-shRNA-NBs were characterized using a TEM (H600, Hitachi, Japan). The average diameters and zeta potential of the NBs, PEI-shRNA, and PEI-shRNA-NBs were analyzed by Dynamic Light Scattering and zeta potential analyzer.

\subsection{Investigation on cell sonoporation}

To see the effects of US exposure upon cell membranes, SMMC-7721 cell was observed with the scanning electron microscope (JEOL JSM-6360LV, Japan). The ultrasound was generated by US system with a single-element 1.0 MHZ frequency transducer. The incubated MMC-7721 cells with PEI-shRNANBs solution were put under US exposure. Different intensity $\left(0,0.3,0.5\right.$ and $\left.1.1 \mathrm{~W} / \mathrm{cm}^{2}\right)$ at a frequency of $1 \mathrm{MHz}$ and pulse repetition frequency of $1 \mathrm{kHz}$ with $50 \%$ of a duty cycle, was applied to the transducer for $60 \mathrm{~s}$ respectively. After US exposure, each sample was fixed, dehydrated and observed with SEM.

\subsection{In vitro cell inhibition and apoptosis evaluation}

There were five groups ( $n=6$ for all groups): (1) DMEM medium containing $10 \%$ FBS; (2) PEIshRNA; (3) PEI-shRNA-NBs; (4) PEI-shRNA with US exposure; (5) PEI-shRNA-NBs with US exposure. The medium of SMMC-7721 cells was changed to $0.2 \mathrm{~mL}$ of the above-mentioned transfer solution and incubated for $10 \mathrm{~min}$. The parameters of US exposure (groups 4 and 5) were as follows: intensity 1.1 $\mathrm{W} / \mathrm{cm}^{2}$ and exposure time $1 \mathrm{~min}$ ). Later on, the medium was altered. After incubation for $48 \mathrm{~h}, 120 \mu \mathrm{L}$ of MTT solution was added and incubated for $4 \mathrm{~h}$. Next, DMSO $(1 \mathrm{~mL})$ was added. The solution was blended till the precipitate was dissolved. The absorbance was measured at $492 \mathrm{~nm}$ using a universal microplate reader and the cell growth inhibition rate (IR) was calculated. The apoptosis rate was identified by flow cytometry. Grouping and processing methods were the same as previously mentioned. Annexin V-FITC $(5 \mu \mathrm{L})$ and propidium iodide (PI, $5 \mu \mathrm{L})$ were added and the samples were analyzed in an hour on a FACSCalibur flow cytometer.

\subsection{In vivo antitumor effect}

Xenograft tumors were induced by the subcutaneous injection of SMMC-7721 cells. There were five groups of 6 each: (1) a negative control group [physiological saline solution]; (2) PEI-shRNA group; (3) PEI-shRNA-NBs group; (4) PEI-shRNA with US exposure group; (5) PEI-shRNA-NBs with US 
Table 1

Physicochemical characteristics

\begin{tabular}{lccc}
\hline Preparation & Diameter $(\mathrm{nm})$ & PI & ZP $(\mathrm{mV})$ \\
\hline NBs & $395.6 \pm 9.6$ & 0.16 & $-19.35 \pm 1.1$ \\
PEI-shRNA & $60.2 \pm 1.8$ & 0.13 & $+35.3 \pm 1.9$ \\
PEI-shRNA-NBs & $382.3 \pm 8.9$ & 0.12 & $+20.3 \pm 1.6$ \\
\hline
\end{tabular}

Abb: PI, polydispersity index; ZP, zeta potential.

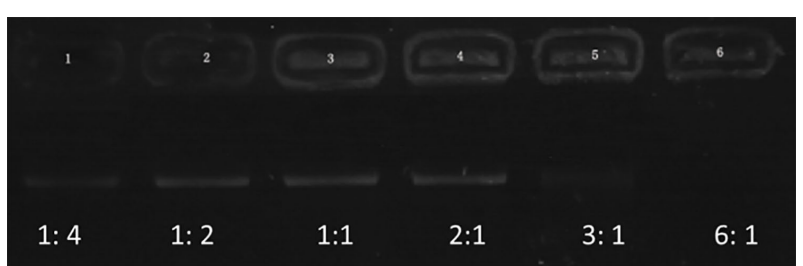

Fig. 1. Electrophoretic mobility of shRNA in agarose gel at various N/P ratios of PEI to shRNA (1:4, 1:2, 1:1, 2:1, 3:1, and $6: 1)$.

exposure group. The PEI-shRNA-NBs containing $6 \mu \mathrm{g}$ of plasmid complexes were prepared and injected through tail veins. The parameters of US exposure (groups 4 and 5) were as follows: intensity of $2.06 \mathrm{~W} / \mathrm{cm}^{2}$ and exposure to low-frequency US for $10 \mathrm{~min}$ at the designated points. We used a commercial clinical ultrasound instrument (Technos MPX; Esaote, Italy) with a linear array transducer to monitor the treatment processing. The xenograft tumor areas were localized before the nanobubbles were injected. With low mechanical index (MI 0.08), the arrival of the nanobubbles into the xenograft tumor areas and the entire treatment process can be visualized. The treatment was repeated every 5 days (20 days in total). Tumor sizes were measured every five days after treatment. All of the mice were euthanized, weighed and then sectioned to hematoxylin-eosin (HE) staining. The rate of inhibition of tumor volume and weight was assessed.

\subsection{Immunohistochemistry}

Tumor sections were used for immunohistochemical studies after undergoing a series of process. Primary antibody against survivin (1:200 dilution in 5\% BSA), bcl-2 (1:100 dilution in 5\% BSA) and bax (1:30 dilution in 5\% BSA) were used. Immunohistochemical processes include dewaxing, washing, incubation, and blocking inactivation of endogenous peroxidase. The tissues were then incubated with primary antibody, washed with PBS, and further incubated with appropriate biotinylated secondary antibody.

\subsection{Statistical analysis}

The data was statistically analyzed with SPSS software (version 18.0, SPSS Inc.).

\section{Results}

\subsection{Preparation of PEI-ShRNA-NBS}

Figure 1 represents the results of agarose gel electrophoresis. A clear DNA band could be seen in each lane with 1:4, 1:2, 1:1, and 2:1 of PEI and shRNA. The band (3:1) was considerably weakening. There 
Table 2

Cell growth inhibition and apoptosis

\begin{tabular}{llcc}
\hline Group & Optical density & Inhibition rate (\%) & Apoptotic cells (\%) \\
\hline a) negative control & $0.982 \pm 0.029^{*}$ & 0 & $0^{*}$ \\
b) PEI-shRNA & $0.833 \pm 0.027^{*}$ & $15.1 \pm 2.75$ & $16.46 \pm 1.08 \%^{*}$ \\
c) PEI-shRNA-NBs & $0.773 \pm 0.009^{*}$ & $21.16 \pm 2.82$ & $19.49 \pm 1.01 \%^{*}$ \\
d) PEI-shRNA with US & $0.716 \pm 0.011^{*}$ & $27.07 \pm 2.26$ & $22.01 \pm 1.52 \%^{*}$ \\
e) PEI-shRNA-NBs with US & $0.541 \pm 0.032^{* \#}$ & $44.92 \pm 3.48$ & $37.1 \pm 2.63 \%^{* \#}$ \\
\hline
\end{tabular}

Notes: ${ }^{*}$ Comparison of experimental groups with the negative control group, $P<0.05$, and

\# comparison of the PEI-shRNA-NBs with US group with other groups, $P<0.05$. Abb: PEI, polyethylenimine; NBs, nanobubbles; US, ultrasound; PEI-shRNA-NBs, NBs bearing PEIshRNA.

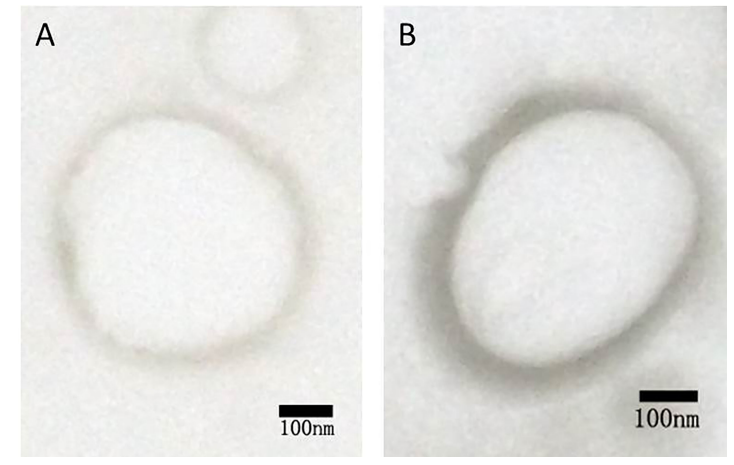

Fig. 2. TEM of (A) unloaded and (B) shRNA-loaded NBs.

was no band to be observed in the lane (6:1). These outcomes recommended that the ratio of $6: 1$ was the optimal proportion of all the plasmids combined with PEI. Both NBs and PEI-shRNA-NBs were spherical with particle size (300 to $500 \mathrm{~nm}$ ). TEM showed the PEI-shRNA-NBs with a well-defined core-shell structure and the shell thickness was about $60 \mathrm{~nm}$ (Fig. 2). Unlike NBs with a fairly smooth surface, the PEI-shRNA-NBs had uneven surface. The average diameter, PI, and zeta potentials of NBs, PEI-shRNA, and PEI-shRNA-NBs were reported in Table 1. Weak positive charge prefers the adhesion of PEI-shRNA-NBs to cancer cells.

\subsection{Investigation on cell sonoporation}

Figure 3 shows SEM images of SMMC 7721cells under different US exposure energy. Normally, the morphology of the cells was spherical and their surfaces were smooth (Fig. 3A). Under the 0.3 and $0.5 \mathrm{~W} / \mathrm{cm}^{2}$ ultrasound energy, conspicuous pits or pores on the cell membranes were not identified. However, when the acoustic-pressure amplitude was increased to $1.1 \mathrm{~W} / \mathrm{cm}^{2}$, rough regions and small pits began to appear on the cell membrane (Fig. 3D). Therefore, proper ultrasonic energy can improve the uptake of the nanocarrier while retaining a good morphology.

\subsection{In vitro cell inhibition and apoptosis evaluation}

The combination of PEI-shRNA-NBs with the US showed the best inhibition compared to the other groups (Table 2). The IRs of the PEI-shRNA and PEI-shRNA-NBs groups were $14.75 \%$ and $20.97 \%$, respectively. After using ultrasound with the same plasmid concentration, the IR increased to $26.85 \%$ and 
Table 3

Volume and mass inhibition of hepatoma

\begin{tabular}{lcccc}
\hline Group & Tumor volume $\left(\mathrm{cm}^{3}\right)$ & Tumor mass $(\mathrm{g})$ & IR of volume (\%) & IR of weight (\%) \\
\hline a) negative control & $4.845 \pm 1.944^{*}$ & $5.713 \pm 1.365^{*}$ & 0 & 0 \\
b) PEI-shRNA & $4.458 \pm 0.764^{*}$ & $4.223 \pm 0.261^{*}$ & 8.0 & 17.5 \\
c) PEI-shRNA-NBs & $3.548 \pm 1.053^{*}$ & $3.485 \pm 0.444^{*}$ & 26.8 & 38.0 \\
d) PEI-shRNA with US & $3.440 \pm 1.014^{*}$ & $3.062 \pm 0.376^{*}$ & 29.0 & 46.4 \\
e) PEI-shRNA-NBs with US & $1.584 \pm 0.513^{* \#}$ & $1.977 \pm 0.247^{* \#}$ & 67.3 & 65.4 \\
\hline
\end{tabular}

Notes: ${ }^{*}$ Comparison of experimental groups with the negative control group, $P<0.05$, and ${ }^{*}$ comparison of the PEIshRNA-NBs with US group with other groups, $P<0.05$.

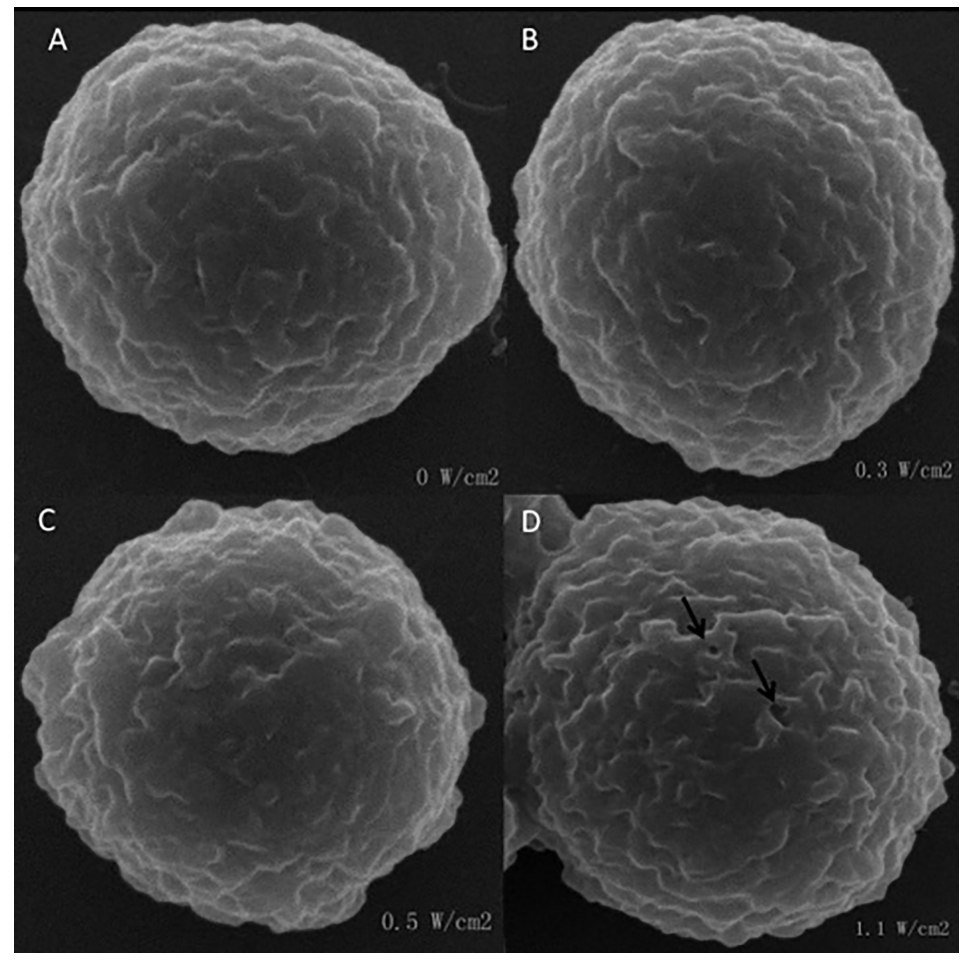

Fig. 3. SEM images of SMMC 7721 cells irradiated at an intensity of $0,0.3,0.5$ and $1.1 \mathrm{~W} / \mathrm{cm}^{2}$ ultrasound.

44.78\%. These data indicate that the PEI-shRNA-NBs combined with US were much more effective in inhibiting the cell proliferation. The number of apoptotic SMMC-7721 cells was observed. PEI-shRNANBs with US group achieved the highest apoptosis level of $37.41 \%$, indicating that a combination of PEI, US, and NBs enhanced shRNA delivery. The percentages of apoptotic cells after treatment with PEI-shRNA-NBs with US, PEI-shRNA with US, PEI-shRNA-NBs, and PEI-shRNA were $37.1 \pm 2.63 \%$, $22.01 \pm 1.52 \%, 19.49 \pm 1.01 \%$, and $16.46 \pm 1.08 \%$ respectively. These results are similar to the MTT data.

\subsection{Antitumor effect in vivo}

After repeated treatments by PEI-shRNA-NBs with US exposure, tumor volume was much smaller than other groups from day 10 and tumor growth was efficiently slowed down (Fig. 4). Consistent with 


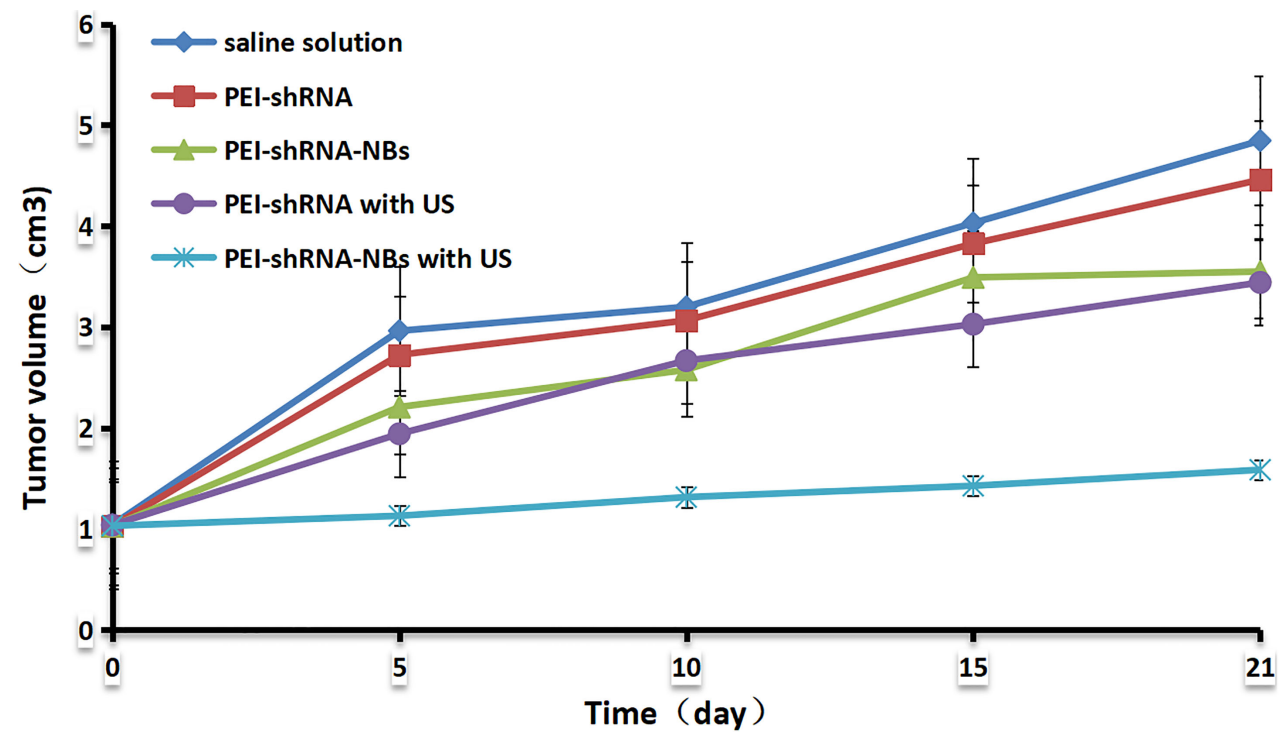

Fig. 4. The tumor growth curves.

our previous in vitro findings, PEI-shRNA-NBs with US group yielded significantly higher volume and weight inhibition rates than the other groups (Table 3 ). In the control group, HE staining showed that the tumor cells actively proliferated and had low differentiation whereas in the treatment group, some tumor tissues showed obvious necrosis and hemorrhage, especially in the PEI-shRNA-NBs plus US group. Immunohistochemical assays of hepatoma sections from mice after the treatments provided further evidence of the expression of the surviving, bcl-2 and bax proteins. Tumor tissues from the PEIshRNA-NBs with US group had the lowest level of surviving and bcl-2 protein and the highest level of bcl-2 protein whereas in the saline groups expression of surviving, bcl-2 and bax were reversed (Fig. 5). These outcomes exhibited that treatment method utilizing the PEI-shRNA-NBs by making use of US exposure could reduce the surviving and bcl-2 expression and to trigger the bax gene very efficiently.

\section{Discussion}

US is a common non-invasive and well-controlled visual tool that is inexpensive and safe for clinical applications. It has sufficient penetration depth to reach the remote target anatomy. Some scholars have studied MBs with US -mediated drug therapy for deep tumors. The study found that the combination of ultrasound, microbubbles and chemotherapy drugs can prolong the quality of life and extend survival in patients with inoperable pancreatic ductal adenocarcinoma [14]. The intimal barrier is a major factor in limiting gene therapy. With the recent developments in the study of micro- as well as nanoparticles, drug delivery has shown great potential for systemic treatment [15]. The cavitation effect caused by USMD enhances the permeability of the cell membrane and cause micro vascular rupture, widening the extracellular space, and the gene can pass through the ruptured microvessels and the extracellular space to the tissue cells for targeted delivery.

Gene therapy for HCC demands non-invasive, efficient, targeted and safe gene transfection strategies. MB combined with US has been used to enhance the efficiency of gene transfection and the ability to target cationic polymer micelles [16]. Nevertheless, MB with larger particle size is not able to penetrate 


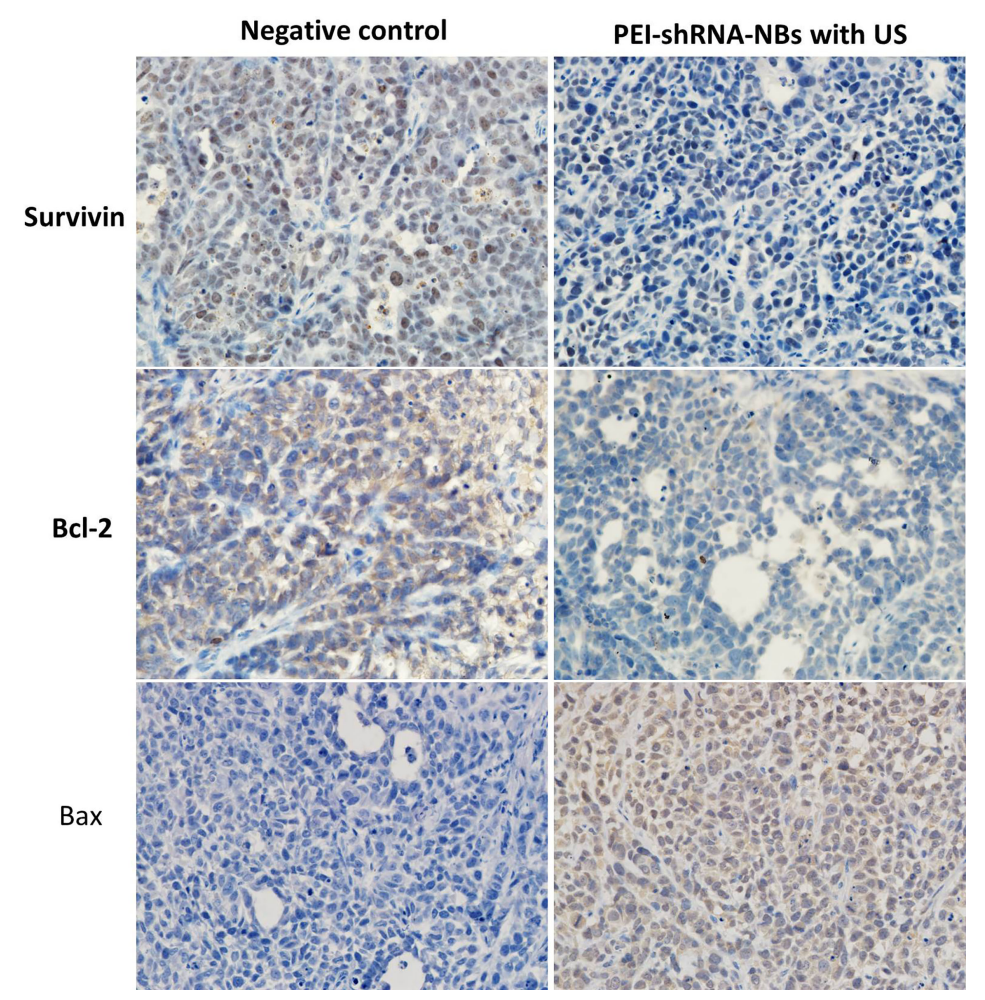

Fig. 5. Immunohistochemical assays of hepatoma sections from mice after the treatments. Brown staining indicated the expression of surviving, bcl-2 or bax protein, and the blue staining showed the nuclei, respectively $(400 \times)$.

the space between vascular endothelial cells. Even though US sonoporation can improve permeability of the vascular endothelium, the membrane permeability of cancer cells continues to be unchanged in the event that MBs cannot approach these cells [17]. Recently, NBs have drawn increasing attention due to their capacity to load DNA into their shell and enhance circulation time. Moreover, it accumulates around cancer cells (via the EPR effect) and beneficial to cellular trafficking.

At present, the application of gene-bearing NBs in inhibiting tumor growth is still in the exploratory stage. Many key issues, such as the preparation of nanobubbles, stability, penetration and transfection efficiency, still require systematic research. Tumor cell lines derived from different tissues can affect ultrasound sonoporation efficiency, and cell membrane permeability can be safely and effectively increased through optimizing exposure parameters [18]. Moreover, unfavorable irradiation parameters can cause cell death [19]. Therefore, it is of great significance to screen the optimal parameters of low frequency ultrasound-mediated gene transfection. In this study, we evaluated the effects of different ultrasound exposures on cell sonication and ultimately optimized the irradiation conditions for transfection. Our previous studies have found that synthetic nanobubbles have greater resistance to cracking under ultrasound energy and longer half-life flushing capabilities in tumor imaging [20] and is more suitable for particular applications that need prolonged viewing time (for example, diagnosis of HCC), which is conducive to the integration of diagnosis and treatment.

PEI as an efficient non-viral vector has been utilized successfully for in vivo [21]. Especially, Deshpande and Mrausinitz [22] documented that the combination of PEI and US can together improve the DNA transfection. In this work PEI-shRNA micelle were used instead of naked shRNA. We also 
examined if ultrasound and PEI possess a synergistic effect to improve shRNA transfection in liver Cancer. Vitro and vivo outcomes demonstrated PEI-shRNA-NBs combined with US therapy could reduce the number of viable liver cancer cells, induce apoptosis in cancer cells and inhibit tumor growth. Histopathological results showed that tumor structures in the PEI-shRNA-NBs with US were destroyed more completely than that of the other groups. The mechanisms involved may be as follows: First, when ultrasound irradiated the tumor area, due to the existence of NB, cavitations appear in the capillaries and tumor tissues, which improved permeability of the vascular endothelium and cancer cell membranes [23]. On the other hand, PEI-shRNA cationic polymers could protect the plasmid from degradation, and the positively charged surface also facilitates their delivery to cancer cells. In addition, gene could be focused on the target site by US regional irradiation.

Survivin, the smallest molecular weight member of apoptosis protein IAP (inhibitor) family, has been demonstrated to play a key role in tumorigenesis [24]. It is highly expressed in liver cancer cell lines and liver cancer tissues, and promotes the rapid deterioration of HCC through its anti-apoptosis, promoting mitosis, proliferation promotion and angiogenesis [25]. These biological functions make Survivin an attractive target for cancer treatment, including HCC treatment [26]. Several independent studies have shown the introduction of survivin siRNA oligonucleotides into the body for the treatment of liver cancer [27]. The bcl-2 proteins adjust a variety of steps in apoptosis as well as obstruct cell death, while pro-apoptotic proteins for instance bax encourage apoptosis [28]. In the study, immunohistochemical method was used to detect apoptosis-related proteins in tumor tissues. The results showed that the inhibition of survivin by management of PEI-shRNA-NBs led to apoptosis induction by down regulating bcl-2 and up regulating of bax.

\section{Conclusion}

In conclusion, we developed a nonviral shRNA gene delivery system utilizing a combination of PEI, US, and NBs for targeting survivin in liver Cancer. The PEI-shRNA-NBs cumulated in the tumor tissue because of the EPR effect. By exposure to the US, micelles shRNA may be released from PEI-shRNANBs in tumor tissues and the shRNA then transmitted efficiently to cancer cells. According to vitro and vivo outcomes, PEI-shRNA-NBs possess great potential to become promising tools of shRNA delivery of targeted cancer therapy.

\section{Acknowledgments}

This work was supported by the Chinese National Natural Science Foundation (81673995), the Research Foundation of Jiangsu Provincial Commission of Health and Family Planning (LGY2016013) and Jiangsu Provincial Medical Youth Talent (QNRC2016643).

\section{Conflict of interest}

None to report. 


\section{References}

[1] Callegari E, Gramantieri L, Domenicali M, et al. MicroRNAs in liver cancer: A model for investigating pathogenesis and novel therapeutic approaches. Cell Death \& Differentiation. 2014; 22(1): 46-57.

[2] Xia Y, Wang C, Xu T, et al. Targeted delivery of HES5-siRNA with novel polypeptide-modified nanoparticles for hepatocellular carcinoma therapy[J]. RSC Advances, 2018; 8(4): 1917-1926.

[3] Guo J, Bourre L, Soden DM, et al. Can non-viral technologies knockdown the barriers to siRNA delivery and achieve the next generation of cancer therapeutics? Biotechnol Adv. 2011; 29(4): 402-417.

[4] de Fougerolles AR. Delivery vehicles for small interfering RNA in vivo. Hum Gene Ther. 2008; 19(2): 125-132.

[5] Liang HD, Tang J, Halliwell M. Sonoporation, drug delivery, and gene therapy. P I Mech Eng H. 2010; 224: 343-361.

[6] Cavalli R, Bisazza A, Trotta M, et al. New chitosan nanobubbles for ultrasound-mediated gene delivery: preparation and in vitro characterization. Int J Nanomed. 2012; 7(14): 3309-3318.

[7] Yu J, Chen Z, Yan F. Advances in Mechanism Studies on Ultrasonic Gene Delivery at Cellular Level[J]. Progress in Biophysics and Molecular Biology, 2018.

[8] Shen J, Zhuo N, Xu S, et al. Resveratrol delivery by ultrasound-mediated nanobubbles targeting nucleus pulposus cells[J]. Nanomedicine. 2018; 13(1).

[9] Horie S, Watanabe Y, Ono M, et al. Evaluation of antitumor effects following tumor necrosis factor $\alpha$ gene delivery using nanobubbles and ultrasound. Cancer Sci. 2011; 102(11): 2082-2089.

[10] Forrest ML, Meister GE, Koerber JT, et al. Partial acetylation of polyethylenimine enhances in vitro gene delivery. Pharm Res-Dordr. 2004; 21(2): 365-371.

[11] Florinas S, Nam HY, Kim SW. Enhanced siRNA Delivery Using a Combination of an Arginine-Grafted Bioreducible Polymer, Ultrasound, and Microbubbles in Cancer Cells. Mol Pharmaceutics. 2013; 10(5): 2021-2030.

[12] Lihua M, Yuan J, Latifa B, et al. Liver cancer initiation is controlled by AP-1 through SIRT6-dependent inhibition of survivin. Nat Cell Biol. 2013; 15(11): 440-440.

[13] Zhang R, Ma LM, Ren J, et al. Survivin knockdown by short hairpin RNA abrogates the growth of human hepatocellular carcinoma xenografts in nude mice. Cancer Gene Ther. 2010; 17(4): 275-288.

[14] Dimcevski G, Kotopoulis S, Bjånes T, et al. A human clinical trial using ultrasound and microbubbles to enhance gemcitabine treatment of inoperable pancreatic cancer[J]. Journal of Controlled Release. 2016; 243: 172-181.

[15] Rahimi-Gorji M, Pourmehran O, Gorji-Bandpy M, et al. CFD simulation of airflow behavior and particle transport and deposition in different breathing conditions through the realistic model of human airways[J]. Journal of Molecular Liquids. 2015; 209: 121-133.

[16] Qiu Y, Luo Y, Zhang Y, et al. The correlation between acoustic cavitation and sonoporation involved in ultrasoundmediated DNA transfection with polyethylenimine (PEI) in vitro. J Control Release. 2010; 145(1): 40-48.

[17] Rapoport N, Gao Z, Kennedy A. Multifunctional nanoparticles for combining ultrasonic tumor imaging and targeted chemotherapy. Jnci Journal Of The National Cancer Institute. 2007; 99(14): 1095-1106.

[18] Shi D, Guo L, Duan S, et al. Influence of tumor cell lines derived from different tissue on sonoporation efficiency under ultrasound microbubble treatment[J]. Ultrasonics Sonochemistry. 2017; 38: 598-603.

[19] $\mathrm{Li} \mathrm{Q}, \mathrm{Li} \mathrm{H}, \mathrm{He} \mathrm{C}$, et al. The use of 5-fluorouracil-loaded nanobubbles combined with low-frequency ultrasound to treat hepatocellular carcinoma in nude mice[J]. European Journal of Medical Research. 2017; 22(1): 48.

[20] Li H, Chen G, Yin L, et al. Ultrasound Enhanced Imaging and Cells Sonoporation of Nanobubbles[J]. Journal of Medical Imaging and Health Informatics. 2016; 6(5): 1227-1232.

[21] Lemkine GF, Goula D, Becker N, et al. Optimisation of polyethylenimine-based gene delivery to mouse brain. J Drug Target. 1999; 7(4): 305-312.

[22] Mangesh CD, Mark RP. Synergistic effect of ultrasound and PEI on DNA transfection in vitro. J Control Release. 2007; 118(1): 126-135.

[23] Figueiredo M, Esenaliev R. PLGA nanoparticles for ultrasound-mediated gene delivery to solid tumors. J Drug Deliv. 2012; 2012: 767839.

[24] Khan Z, Khan AA, Yadav H, Prasad G, Bisen PS. Survivin, a molecular target for therapeutic interventions in squamous cell carcinoma, Cell. Mol. Biol. Lett. 2017; 22: 8.

[25] Han Y, Wu Y, Yang C, et al. Dynamic and specific immune responses against multiple tumor antigens were elicited in patients with hepatocellular carcinoma after cell-based immunotherapy[J]. Journal of Translational Medicine. 2017; 15(1): 64.

[26] Ryan BM, O’Donovan N, Duffy MJ. Survivin: a new target for anti-cancer therapy[J]. Cancer Treatment Reviews. 2009; 35(7): 553-562.

[27] Wu Z, Xu XL, Zhang JZ, et al. Magnetic cationic amylose nanoparticles used to deliver survivin-small interfering RNA for gene therapy of hepatocellular carcinoma in vitro[J]. Nanomaterials. 2017; 7(5): 110.

[28] Hinnis AR, Luckett JCA, Walker RA. Survivin is an independent predictor of short-term survival in poor prognostic breast cancer patients. Brit J Cancer. 2007; 96(4): 639-645. 